Published in final edited form as:

Chem Res Toxicol. 2019 June 17; 32(6): 982-985. doi:10.1021/acs.chemrestox.9b00171.

\title{
Common E-Cigarette Flavoring Chemicals Impair Neutrophil Phagocytosis and Oxidative Burst
}

\author{
Elise Hickman ${ }^{\dagger, \ddagger}$, Carolina A. Herrera ${ }^{\S}$, and Ilona Jaspers ${ }^{\star}, \dagger, \ddagger$ \\ tCurriculum in Toxicology \& Environmental Medicine, The University of North Carolina at Chapel \\ Hill, Chapel Hill, North Carolina 27514, United States \\ ‡Center for Environmental Medicine, Asthma, and Lung Biology, The University of North Carolina \\ at Chapel Hill, Chapel Hill, North Carolina 27514, United States \\ §Department of Pathology and Laboratory Medicine, The University of North Carolina at Chapel \\ Hill, Chapel Hill, North Carolina 27514, United States
}

\begin{abstract}
E-cigarette flavorings have not been thoroughly evaluated for inhalational toxicity. We have shown that the flavoring chemical cinnamaldehyde impairs human neutrophils, macrophages, and natural killer cells. Here we investigated the effects of other common e-liquid flavoring chemicals on phagocytosis and oxidative burst in neutrophils. We demonstrate that cinnamaldehyde and ethyl vanillin dose-dependently decrease oxidative burst and that benzaldehyde and benzaldehyde propylene glycol acetal dose-dependently impair phagocytosis. Isoamyl acetate did not affect either measure of neutrophil function. These data suggest that inhaling aromatic aldehydic flavoring chemicals, such as cinnamaldehyde, benzaldehyde, benzaldehyde propylene glycol acetal, or ethyl vanillin, could impair neutrophil function.
\end{abstract}

\section{Graphical Abstract}

\footnotetext{
*Corresponding Author: ilona_jaspers@med.unc.edu. Supporting Information

The Supporting Information is available free of charge on the ACS Publications website at DOI: 10.1021/acs.chemrestox.9b00171. Overall study demographics; maximum OCR for each flavoring chemical; time to maximum OCR for each flavoring chemical; maximum ECAR for each flavoring chemical; time to maximum ECAR for each flavoring chemical; IC50 values for neutrophil oxidative burst AUC and phagocytosis; neutrophil oxidative burst rate graphs; neutrophil glycolysis during oxidative burst (PDF) The authors declare no competing financial interest.
} 


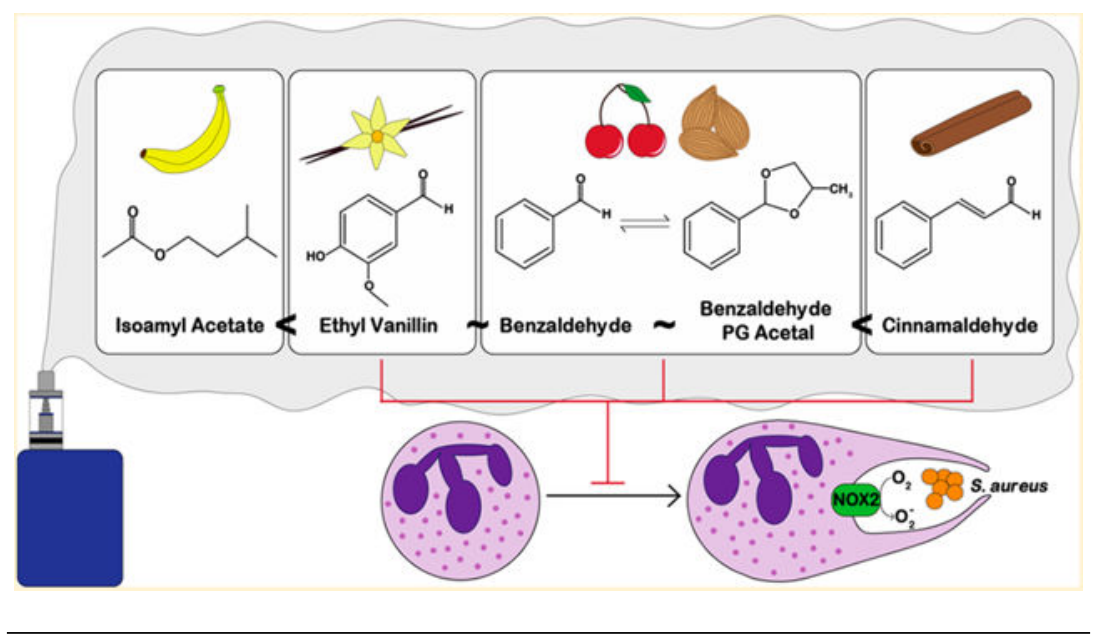

The growing popularity of e-cigarettes has been linked to the public opinion and promotion of them as a safer alternative to tobacco cigarettes ${ }^{1}$ and some studies suggesting e-cigarettes help in smoking cessation. ${ }^{2}$ Of particular concern is adolescent use of e-cigarettes. Currently, $20.8 \%$ of high school students in the US are e-cigarette users. ${ }^{3}$ E-cigarettes heat and aerosolize e-liquids, which are typically composed of humectants (propylene glycol (PG) and vegetable glycerin), nicotine, and flavoring chemicals. The wide variety of e-liquid flavors is one of the primary reasons that using e-cigarettes is so appealing to adolescents, ${ }^{4,5}$ yet many of these flavoring chemicals have not been evaluated for inhalational toxicity. ${ }^{6}$ Hence, understanding the potential toxicity of e-cigarette flavorings is necessary to inform regulation of e-liquid manufacturing and to educate the public about e-cigarette safety.

Neutrophils, along with airway macrophages, are one of the body's first lines of defense against inhaled pathogens, ${ }^{7}$ making them an important target for inhaled toxicants. There is emerging appreciation for the complex interplay between cellular metabolism and innate immune response as well as the concept that bioenergetic changes are at the center of innate immune dysfunction and immune cell phenotypes. ${ }^{8}$ However, bioenergetic characterization of the innate immune system has been limited primarily to macrophages, while neutrophils have been largely ignored. ${ }^{9,10}$ Furthermore, the ability of toxicants to alter neutrophil bioenergetics and downstream functions remains unclear. Our lab has demonstrated that the flavoring chemical cinnamaldehyde significantly affects cellular bioenergetics, which in turn causes impairment of key innate immune functions of respiratory epithelial cells. ${ }^{11}$ Hence, disruption of cellular bioenergetics could be a central mechanism mediating adverse effects of flavoring chemicals.

In this study, we investigated the effects of four common flavoring chemicals, cinnamaldehyde (cinnamon), ethyl vanillin (vanilla), benzaldehyde (almond or cherry), and isoamyl acetate (banana), on neutrophil oxidative burst and phagocytosis. Because we had previously observed impairment of airway and immune cell function by cinnamaldehyde, 11,12 we were interested in whether other common aromatic aldehydes found in e-cigarettes also impaired cellular function. ${ }^{13-15}$ We chose isoamyl acetate, an ester that is commonly detected in e-cigarettes, ${ }^{15}$ for comparison. We also assessed the effects of benzaldehyde PG 
acetal, which can form via the reaction of benzaldehyde and PG in e-liquids ${ }^{16}$ and has been identified by multiple research groups in e-liquids and e-cigarette aerosols. ${ }^{13-15}$

We isolated neutrophils from venous blood of healthy human subjects as described previously (Table S1). ${ }^{12}$ The effect of flavoring chemicals (Sigma-Aldrich, ST. Louis, MO, USA) on neutrophil oxidative burst was measured via sequential injection of a flavoring compound ( $0-5 \mathrm{mM}$ final concentration) followed by injection of protein kinase $\mathrm{C}$ agonist phorbol 12-myristate 13-acetate (PMA, $100 \mathrm{ng} / \mathrm{mL}$ final concentration) on a Seahorse XFe24 Extracellular Flux Analyzer (Agilent Technologies, Santa Clara, CA, USA) at $37{ }^{\circ} \mathrm{C}$ (Figure 1A). Concentrations between $0-5 \mathrm{mM}$ were used because this is approximately two orders of magnitude below maximum concentrations of these flavorings in e-liquids, and we have observed cellular effects on other cell types in this concentration range. ${ }^{12-15}$ The total oxygen consumption during oxidative burst represents the amount of oxygen converted by the neutrophils to superoxide. This was quantified by calculating the area under the curve after PMA stimulation using oxygen consumption rate (OCR, pmol/min) data (Figure 1, Figure S1). Extracellular acidification rate (ECAR, $\mathrm{mpH} / \mathrm{min}$ ), a measure of glycolysis, was simultaneously measured with OCR during these experiments.

Cinnamaldehyde and ethyl vanillin decreased total oxygen consumption in a dose-dependent manner (Figure 1B,C), with a calculated $\mathrm{IC}_{50}$ of $0.39 \pm 0.04 \mathrm{mM}$ and $0.42 \pm 0.1 \mathrm{mM}$, respectively. Benzaldehyde and benzaldehyde $\mathrm{PG}$ acetal significantly decreased oxygen consumption only at the highest dose, $5 \mathrm{mM}$ (Figure 1D,E). Isoamyl acetate did not affect oxygen consumption during oxidative burst (Figure 1F).

Using the area under the curve analysis, we also obtained the maximum OCR, time to the maximum OCR, and time to baseline OCR. The data for the maximum OCR followed similar trends to those of the total oxygen consumption data (Table S2). The highest doses of ethyl vanillin and benzaldehyde $(5 \mathrm{mM})$ significantly decreased the time to maximum OCR $(p<0.05$, Table S3). There was no significant difference in the time it took to return to baseline for any of the doses of the flavoring chemicals (data not shown). The ECAR data mirror the OCR data, as expected (Figure S2, Tables S4 and S5).

Next, we determined the effect of e-cigarette flavoring chemicals on neutrophil phagocytosis. Neutrophils plated in a 96-well plate were challenged in triplicate with $0-5$ $\mathrm{mM}$ of flavoring chemical for $1 \mathrm{~h}$ at $37^{\circ} \mathrm{C}$, and phagocytosis was measured using pHrodo Red Staphylococcus aureus BioParticles (Thermo Fischer Scientific, Waltham, MA, USA) as described previously. ${ }^{12}$ Cinnamaldehyde $(\mathrm{CA}, 1 \mathrm{mM})$ was used as a positive control for inhibition of phagocytosis. ${ }^{12}$

We have previously shown that cinnamaldehyde impairs neutrophil phagocytosis. ${ }^{12}$ Here, we show that other aromatic aldehydes are also capable of impairing neutrophil phagocytosis. Ethyl vanillin, benzaldehyde, and benzaldehyde PG acetal significantly decreased neutrophil phagocytosis (Figure 2A/B/C), while isoamyl acetate (Figure 2D) had no effect. The inhibition caused by benzaldehyde $\mathrm{PG}$ acetal was more potent than that observed with benzaldehyde, with $\mathrm{IC}_{50}$ values of $4.72 \pm 2.05$ and $1.89 \pm 0.66 \mathrm{mM}$ for benzaldehyde and benzaldehyde PG acetal, respectively, suggesting that chemicals formed 
via the reaction of flavoring chemicals and e-liquid constituents under ambient conditions may exert stronger toxicological effects than those of the base favoring chemical.

While there are thousands of different commercially available flavored e-liquids, ${ }^{17}$ they are created using a much smaller set of flavoring chemicals that are combined to create unique flavors. ${ }^{13-15}$ Chemical analyses of flavored e-liquids and e-cigarette aerosols from separate studies reveal that many different flavored e-liquids share common flavoring chemicals and that these flavoring chemicals are present in e-liquids at up to molar concentrations. ${ }^{13-15}$ Interestingly, the flavoring chemicals we found to impair neutrophil function share a common chemical class: they are all aromatic aldehydes or their derivatives.

Though they share a common chemical class, these flavorings have unique functional groups, which may explain their differential activities. For example, cinnamaldehyde is an $a, \beta$-unsaturated aldehyde that can covalently bind and modify thiols, including interactions with cysteinyl groups on proteins, ${ }^{18}$ whereas vanillin (the sister compound to ethyl vanillin) has been shown to interact with proteins via formation of Schiff bases. ${ }^{19}$ Benzaldehyde shares structural similarity to cinnamaldehyde but lacks the reactive $a, \beta$-unsaturated moiety, which may explain why it inhibited neutrophil function to a lesser extent than cinnamaldehyde. Potential mechanisms underlying decreased oxidative burst observed here could include direct inhibition of key proteins in glycolysis or the pentose phosphate pathway via thiol modification, alteration of glucose uptake, or interference with NADPH oxidase complex formation and activation. The bioenergetic effects of these chemicals could also have implications for assays such as the MTT assay, which relies on mitochondrial metabolism as an indicator of cytotoxicity.

Erythropel et al. recently demonstrated that aldehydic flavoring chemicals such as cinnamaldehyde and benzaldehyde can react with PG under ambient conditions to form flavorant PG acetals, ${ }^{16}$ which have been reported in e-liquids and e-cigarette aerosols. ${ }^{13-15}$ Here, we demonstrated that benzaldehyde PG acetal can impair phagocytosis more potently than benzaldehyde (Figure 2B,C). However, benzaldehyde impaired oxidative burst slightly more potently than benzaldehyde PG acetal (Figure 1D,E). These results further support the previously published notion that flavoring chemicals present in e-liquids can also form secondary or tertiary reaction products through interactions with various components of the e-liquid, which alter their biological activities and toxicities.

Among the most challenging components of in vitro experimental research models is estimating physiologically relevant doses of inhaled flavoring chemicals. The flavoring chemicals we studied have been reported in e-liquids in hundreds of millimolar to molar concentrations, ${ }^{12,14,15,20}$ and these flavoring chemicals carry over to aerosol with high efficiency. ${ }^{16,20}$ Our highest doses are two orders of magnitude below these ranges; however, because human in vivo exposure has not been precisely quantified, it is difficult to compare the doses at which we found effects to the level of exposure in e-cigarette users.

In addition, our results show differing potencies and effects of the flavoring chemicals on oxidative burst and phagocytosis (Table S6). This discrepancy may be due to the method of neutrophil activation used. Uptake of $S$. aureus BioParticles more accurately recapitulates 
neutrophil phagocytosis in vivo, while stimulation of neutrophils with PMA directly activates protein kinase $\mathrm{C}$, in the absence of a relevant pathogen.

Overall, our data demonstrate that flavoring chemicals present in e-liquids can impair neutrophil function at different levels. Impaired neutrophil function has been shown to play a role in decreased bacterial clearance, especially in vulnerable patients such as those with chronic obstructive pulmonary disease. ${ }^{21}$ Thus, inhalation of e-cigarette flavorings, especially aldehydes, could significantly impair neutrophil function and consequently increase susceptibility to infection and respiratory disease. Since we found that chemicals from the same class (e.g., aromatic aldehydes) may affect immune cell function in a similar manner, assessing the toxicity of flavoring chemicals in e-cigarettes could be done by chemical class rather than by individual compound.

\section{Supplementary Material}

Refer to Web version on PubMed Central for supplementary material.

\section{ACKNOWLEDGMENTS}

The authors would like to thank Carole Robinette, Martha Almond, and Brian Ring for coordination of study subjects; Dr. James Samet, Dr. Katelyn Lavrich, and Elizabeth Corteselli for use of and training on the Seahorse machine; and Dr. Meghan Rebuli for the feedback on the manuscript.

Funding

This research was funded by National Institutes of Health (NIH) Grant Nos. R01 HL139369 and T32 ES007126.

\section{ABBREVIATIONS}

$\begin{array}{ll}\text { CA } & \text { cinnamaldehyde } \\ \text { ECAR } & \text { extracellular acidification rate } \\ \text { NADPH } & \text { nicotinamide adenine dinucleotide phosphate hydrogen } \\ \text { OCR } & \text { oxygen consumption rate } \\ \text { PG } & \text { propylene glycol } \\ \text { PMA } & \text { phorbol 12-myristate 13-acetate }\end{array}$

\section{REFERENCES}

(1). Amrock SM, Zakhar J, Zhou S, and Weitzman M (2015) Perception of E-Cigarette Harm and Its Correlation With Use Among U.S. Adolescents. Nicotine Tob. Res 17 (3), 330-336. [PubMed: 25125321]

(2). Hajek P, Phillips-Waller A, Przulj D, Pesola F, Myers Smith K, Bisal N, Li J, Parrott S, Sasieni P, Dawkins L, Ross L, Goniewicz M, Wu Q, and McRobbie HJ (2019) A Randomized Trial of ECigarettes versus Nicotine-Replacement Therapy. N. Engl. J. Med 380 (7), 629-637. [PubMed: 30699054]

(3). Wang TW, Gentzke A, Sharapova S, Cullen KA, Ambrose BK, and Jamal A (2018) Tobacco Product Use Among Middle and High School Students — United States, 2011-2017. MMWR Morb Mortal Wkly Rep 67, 629-633. [PubMed: 29879097] 
(4). Jongenelis MI, Kameron C, Brennan E, Rudaizky D, Slevin T, and Pettigrew S (2018) E-cigarette product preferences among Australian young adult e-cigarette users. Aust N Z. J. Public Health 42 (6), 572-574. [PubMed: 30248716]

(5). Audrain-McGovern J, Strasser AA, and Wileyto EP (2016) The impact of flavoring on the rewarding and reinforcing value of e-cigarettes with nicotine among young adult smokers. Drug Alcohol Depend. 166, 263-7. [PubMed: 27426010]

(6). Safety Assessment and Regulatory Authority to Use Flavors: Focus on Electronic Nicotine Delivery Systems and Flavored Tobacco Products; FEMA, 2016.

(7). Mócsai A (2013) Diverse novel functions of neutrophils in immunity, inflammation, and beyond. J. Exp. Med 210 (7), 1283-1299. [PubMed: 23825232]

(8). Weinberg SE, Sena LA, and Chandel NS (2015) Mitochondria in the regulation of innate and adaptive immunity. Immunity 42 (3), 406-17. [PubMed: 25786173]

(9). O’Neill LA, Kishton RJ, and Rathmell J (2016) A guide to immunometabolism for immunologists. Nat. Rev. Immunol 16 (9), 553-65. [PubMed: 27396447]

(10). Lavrich KS, Speen AM, Ghio AJ, Bromberg PA, Samet JM, and Alexis NE (2018) Macrophages from The Upper and Lower Human Respiratory Tract Are Metabolically Distinct. Am. J. Physiol Lung Cell Mol. Physiol 315, L752. [PubMed: 30091382]

(11). Clapp PW, Lavrich KS, van Heusden C, Lazarowski ER, Carson JL, and Jaspers I (2019) Cinnamaldehyde in Flavored E-Cigarette Liquids Temporarily Suppresses Bronchial Epithelial Cell Ciliary Motility by Dysregulation of Mitochondrial Function. Am. J. Physiol Lung Cell Mol. Physiol 316, L470. [PubMed: 30604630]

(12). Clapp PW, Pawlak EA, Lackey JT, Keating JE, Reeber SL, Glish GL, and Jaspers I (2017) Flavored e-cigarette liquids and cinnamaldehyde impair respiratory innate immune cell function. Am. J. Physiol Lung Cell Mol. Physiol 313 (2), L278-L292. [PubMed: 28495856]

(13). Peace MR, Mulder HA, Baird TR, Butler KE, Friedrich AK, Stone JW, Turner JBM, Poklis A, and Poklis JL (2018) Evaluation of Nicotine and the Components of e-Liquids Generated from eCigarette Aerosols. J. Anal. Toxicol 42 (8), 537-543. [PubMed: 30371842]

(14). Tierney PA, Karpinski CD, Brown JE, Luo W, and Pankow JF (2016) Flavour chemicals in electronic cigarette fluids. Tob Control 25 (e1), e10-5. [PubMed: 25877377]

(15). Omaiye EE, McWhirter KJ, Luo W, Tierney PA, Pankow JF, and Talbot P (2019) High concentrations of flavor chemicals are present in electronic cigarette refill fluids. Sci. Rep 9 (1), 2468. [PubMed: 30792477]

(16). Erythropel HC, Jabba SV, DeWinter TM, Mendizabal M, Anastas PT, Jordt SE, and Zimmerman JB (2018) Formation of flavorant-propylene Glycol Adducts With Novel Toxicological Properties in Chemically Unstable E-Cigarette Liquids. Nicotine Tob. Res, nty192.

(17). Zhu SH, Sun JY, Bonnevie E, Cummins SE, Gamst A, Yin L, and Lee M (2014) Four hundred and sixty brands of e-cigarettes and counting: implications for product regulation. Tob Control 23 (Suppl 3), iii3-9. [PubMed: 24935895]

(18). Weibel H, and Hansen J (1989) Interaction of cinnamaldehyde (a sensitizer in fragrance) with protein. Contact Dermatitis 20 (3), 161-6. [PubMed: 2721181]

(19). Chobpattana W, Jeon IJ, and Smith JS (2000) Kinetics of interaction of vanillin with amino acids and peptides in model systems. J. Agric. Food Chem 48 (9), 3885-9. [PubMed: 10995286]

(20). Behar RZ, Luo W, McWhirter KJ, Pankow JF, and Talbot P (2018) Analytical and toxicological evaluation of flavor chemicals in electronic cigarette refill fluids. Sci. Rep 8 (1), 8288. [PubMed: 29844439]

(21). Stockley JA, Walton GM, Lord JM, and Sapey E (2013) Aberrant neutrophil functions in stable chronic obstructive pulmonary disease: the neutrophil as an immunotherapeutic target. Int. Immunopharmacol 17 (4), 1211-7. [PubMed: 23994347] 
A

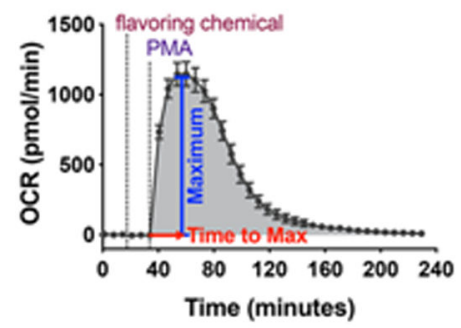

C

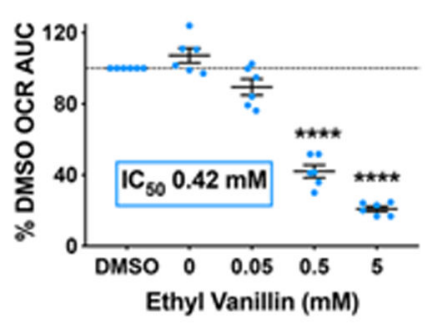

E

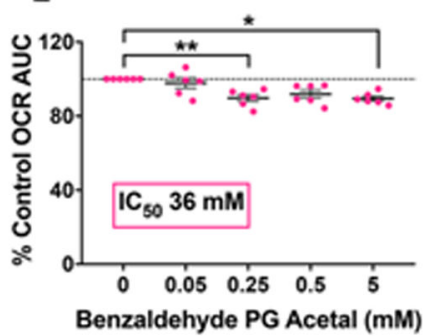

B

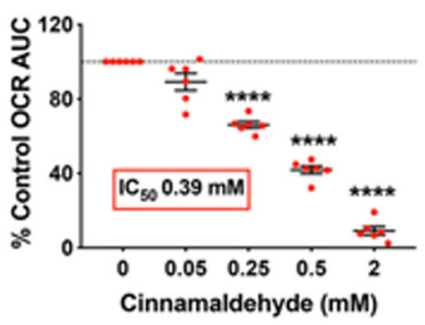

D

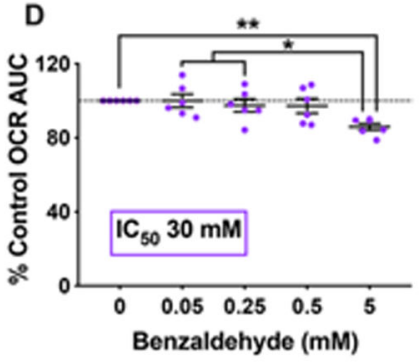

$\mathbf{F}$

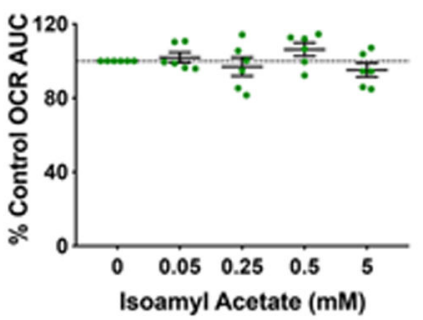

Figure 1.

Neutrophil oxidative burst. (A) Seahorse Extracellular Flux assay was used to measure the effects of flavoring chemicals (B) cinnamaldehyde, $(\mathrm{C})$ ethyl vanillin, (D) benzaldehyde, $(\mathrm{E})$ benzaldehyde PG acetal, (F) and isoamyl acetate on PMA-stimulated neutrophil oxidative burst. Oxygen consumption rate (OCR, $\mathrm{pmol} / \mathrm{min}$ ) was used to determine total oxygen consumption by integrating the oxygen consumption rate (OCR, pmol/min) of PMAstimulated neutrophils over time (area under the curve, AUC). Data represents mean \pm SEM with $n=6$.

$* p<0.05, * * p<0.01, * * * p<0.001, * * * * p<0.0001$. 
A
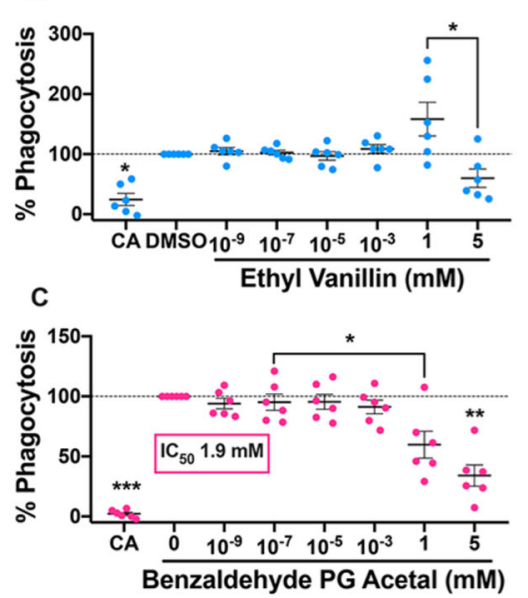

B

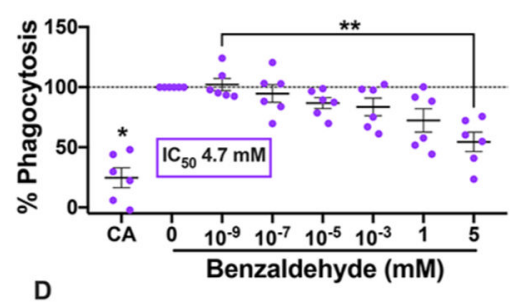

D

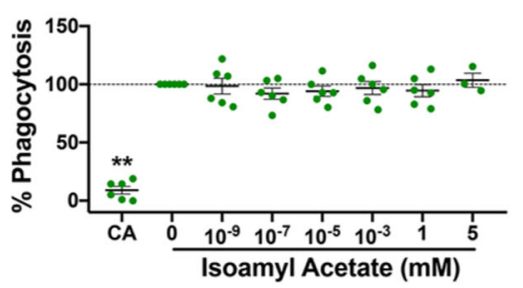

Figure 2.

Neutrophil phagocytosis. Effects of flavoring chemicals (A) ethyl vanillin, (B) benzaldehyde, (C) benzaldehyde PG acetal, and (D) isoamyl acetate on neutrophil phagocytosis as assessed with pHrodo red $S$. aureus BioParticles. Data represent mean \pm SEM with $n=6$, with the exception of $n=3$ for $5 \mathrm{mM}$ isoamyl acetate.

$* p<0.05$, ** $p<0.01$. 